Check for updates

Cite this: RSC Adv., 2018, 8, 27863

\title{
Development of a candidate reference sample for the characterization of tip-enhanced Raman spectroscopy spatial resolution $\uparrow$
}

\author{
Alessio Sacco, (D) ab Dario Imbraguglio, ${ }^{a}$ Andrea M. Giovannozzi, ${ }^{a}$ Chiara Portesi ${ }^{\star a}$ \\ and Andrea M. Rossia
}

Tip-Enhanced Raman Spectroscopy (TERS) is a topographic and chemical analysis technique with nanoscale resolution, consisting of the combination of Scanning Probe Microscopy (SPM) and Localized Surface Plasmon Resonance (LSPR) for the enhancement of Raman scattering in the vicinity of the probe. The quantification of spatial resolution represents an important issue, and, as of now, standards for calibration are not available. In the present work a candidate reference sample for TERS measurements was fabricated. It consists of a flat, conductive gold surface with a nanometric grating of a self-assembled monolayer of Raman-active organic molecules fabricated by an optimized Electron Beam Lithography (EBL) method to replicate established SPM calibration standards. Its feasibility as a TERS standard was tested by STM-TERS imaging.

Received 2nd May 2018

Accepted 30th July 2018

DOI: $10.1039 / \mathrm{c} 8 \mathrm{ra03762k}$

rsc.li/rsc-advances

In SPM, a tip with a sharp apex allows probing of areas a few

\section{Introduction}

Industry and scientific research rely upon the ability to determine quantities accurately and unequivocally and, to achieve that, establishing common guidelines for measurements and reference samples for calibration is important. Metrology tackles this issue to provide the basis for a single, coherent system of measurements throughout the world, traceable to the International System of Units (SI), with the respective universal practical realisation of the units and methods of measurement.

Raman spectroscopy is an analytical technique with increasing industrial applications, but it currently lacks reference materials. The application of certified reference materials (CRMs) in analytical chemistry for quality control purposes is well recognized and recommended by a wide range of international, national and professional organizations. In this work we propose a fabrication method for a candidate reference sample for spatial resolution calibration of Tip-Enhanced Raman spectroscopy (TERS) setups. TERS combines plasmon-enhanced Raman spectroscopy and scanning probe microscopy (SPM), a technique which has been developed to measure spatial features of surfaces with nanometric resolution, allowing simultaneous chemical and topographic mapping of surfaces with nanometric resolution. ${ }^{\mathbf{1 - 5}}$

Istituto Nazionale di Ricerca Metrologica, Torino, 10135, Italy.E-mail: c.portesi@ inrim.it

${ }^{b}$ Politecnico di Torino, Torino, 10129, Italy

$\dagger$ Electronic supplementary information (ESI) available. See DOI: $10.1039 / \mathrm{c} 8 \mathrm{ra} 03762 \mathrm{k}$ nanometres wide and the reconstruction of topographic images with spatial resolution much higher than conventional optical microscopy. Indeed, the latter is diffraction-limited, making it impossible to resolve features smaller than approximately half of the light wavelength (for visible light, no less than about 200 $\mathrm{nm}$ ), while SPM can achieve atomic spatial resolution depending on the characteristics of the probe, the feedback and the apparatus employed.

In a TERS setup, a metallic SPM tip is illuminated by a laser beam. The emitted signal is collected by a microscope objective and analysed by a Raman spectrophotometer. By combining the two techniques, it is possible to register topographic and chemical maps, each with a spatial resolution (usually quantified by the ability to spatially resolve two adjacent targets, or by calculating the FWHM of the profile of a sharp feature in the image) dictated by the probe apex and comparable to its nanometric dimensions. ${ }^{6-11}$ To achieve TERS, the surface plasmons on the probe tips must resonate with the excitation laser electromagnetic frequency so that a plasmonically enhanced, Ramanamplifying electromagnetic field is formed, and is localised in the vicinity of the tip apex. ${ }^{1}$ This physical phenomenon is the same as in surface-enhanced Raman spectroscopy, but in TERS the Raman signal amplification is bound to a single, restricted region (a hotspot), from which the SPM feedback also arises. Raman-amplifying SPM probes may be fabricated by either coating a classical SPM probe with an appropriate metal, or by shaping a probe from solid metal such as a wire (silver or gold are widespread choices for visible excitation wavelengths).

Even though in the literature many significant results have been obtained by exploiting TERS capabilities, including single- 
a)

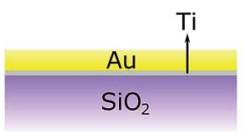

b)

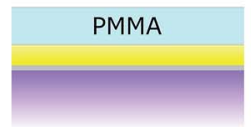

c) $\mathrm{EBL} \quad \mathrm{EBL}$

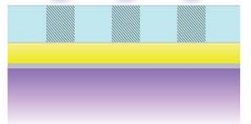

d)

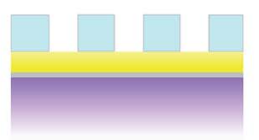

e)

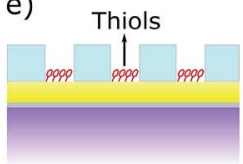

f)

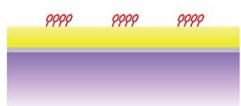

Fig. 1 Scheme of the steps of the standard production process. The initial flat $20 \mathrm{~nm}$ Au surface (a), deposited on $\mathrm{SiO}_{2}$ with a Ti adhesion layer, is covered by a $50 \mathrm{~nm}$ PMMA mask (b), which is then selectively exposed to EBL (c). After the mask development, bare Au areas remain (d), where thiols can selectively bond. The surface is soaked in a thiol solution until a self-assembled monolayer is formed (e), then rinsed, and sonicated in acetone to remove the mask. After this process, the gold surface with a nanopatterned thiol SAM is ready (f).

molecule and ultrafast mapping, and TERS has been applied to diverse environments such as ultrahigh vacuum and liquids, ${ }^{\mathbf{1 2 - 1 4}}$ one of the most urgent issues is related to the questions about reproducibility of the results and the standardization of the methods. Actually, while metrological SPMs have been already developed, the way towards metrological TERS has yet to be paved. By metrological SPM, it is possible to trace three-dimensional length measurements to the length SI standard with nanometric accuracy. ${ }^{\mathbf{1 5 , 1 6}}$ By metrologically calibrated TERS, the chemical information will be traceable to the SI as well and, at the same time, the measurements will gain in terms of reproducibility.

In this paper, a candidate sample suitable to achieve TERS chemical imaging traceability to the metre by means of an SPMbased calibration procedure for nanometric accuracy is presented. It is designated to be both highly thermally and electrically conductive to minimise radiation heating and for measurements with both force-based and tunnelling current TERS feedbacks, making it a suitable candidate to be a universal TERS standard for calibration and comparisons, regardless of the apparatus specifications. ${ }^{17}$ Its gold surface, albeit flat, further enhances TERS signals, leading to measurements in gap-mode, resulting in much more intense signals than those arising from a non-gap mode TERS measurement, ${ }^{18}$ and a much lower detection limit. ${ }^{19}$ The sample is characterised during the different steps of the production process with several techniques, including confocal Raman, AFM, and TERS imaging in STM feedback.

\section{Experimental}

\section{Sample}

The sample was manufactured as described in Fig. 1, starting from a clean $\mathrm{SiO}_{2} / \mathrm{Si}$ surface $\left(280 \mathrm{~nm} \mathrm{SiO}_{2}\right.$ on monocrystalline $\mathrm{Si}$, by Si-Mat). $20 \mathrm{~nm}$ of gold (99.999\%, Alfa Aesar) were deposited by electron-gun evaporation at a rate of $0.1 \mathrm{~nm} \mathrm{~s}^{-1}$ after the deposition of $5 \mathrm{~nm}$ of titanium (99.99\%, Alfa Aesar) as adhesion layer. The resulting surface was then covered by $50 \mathrm{~nm}$ of poly methylmethacrylate (PMMA) by spin-coating (at $6000 \mathrm{rpm}$ for $60 \mathrm{~s}$ ) to form the electron-sensitive layer. The Electron Beam Lithography (EBL) was performed with a dose of $670 \mu \mathrm{C} \mathrm{cm}^{-2}$ and an acceleration voltage of $30 \mathrm{kV}$. Afterwards, it was developed in methyl isobutyl ketone (MIBK in isopropyl alcohol $1: 3$ ) for $30 \mathrm{~s}$, then rinsed in IPA and water and dried with nitrogen. The nanostructured samples were then soaked either in thiophenol solution (TP, $\geq 99 \%$ from Sigma-Aldrich, $5 \mathrm{mM}$ in ethanol) or in 7- mercapto-4-methylcoumarin (MMC, $\geq 97 \%$ from Sigma-Aldrich) solution ( $1 \mathrm{mM}$ in ethanol) for $18 \mathrm{~h}$, resulting in the formation of thiol Self-Assembled Monolayers (SAM) ${ }^{20,21}$ selectively bonded on the exposed Au portion of the sample. The specimens were then thoroughly rinsed in ethanol and dried with nitrogen. At the end, to remove the PMMA resist, the samples were sonicated in acetone ( $\geq 99.8 \%$, Carlo Erba) for 5 minutes $\left(37 \mathrm{kHz}\right.$ at $\left.30{ }^{\circ} \mathrm{C}\right)$, rinsed with ethanol and nitrogen flux dried.

\section{Apparatus}

TERS measurements were performed in ambient conditions and in STM feedback with a NT-MDT Ntegra scanning probe microscope, equipped with a $10 \mu \mathrm{m} \times 10 \mu \mathrm{m}$ piezoelectric scanner and customised with mirrors to allow tip illumination and scanning of the Raman laser excitation source (He-Ne $633 \mathrm{~nm}$, electrically pumped, continuous, linearly polarized) from a modified Thermo DXR spectrometer, responsible for the spectra acquisition as well. The system achieves $5 \mathrm{~cm}^{-1}$ spectral resolution in the $50-3600 \mathrm{~cm}^{-1}$ spectral range. Illumination of the tip and signal collection is mediated by a $100 \times$, long working distance microscope objective (Mitutoyo M Plan Apo $100,0.7 \mathrm{NA}$ ) in a top illumination configuration (Fig. 2). ${ }^{22}$ The TERS tips were fabricated by electrochemical etching of a $0.25 \mathrm{~mm}$ diameter $\mathrm{Ag}$ wire (99.99\% purity, from SigmaAldrich) in a $1: 2 \mathrm{v} / \mathrm{v}$ solution of perchloric acid $\left(70 \% \mathrm{HClO}_{4}\right.$ in water, from Merck) in methanol (special grade, by Carlo Erba) by applying a $(8.1 \pm 0.1) \mathrm{V}$ voltage difference with respect to

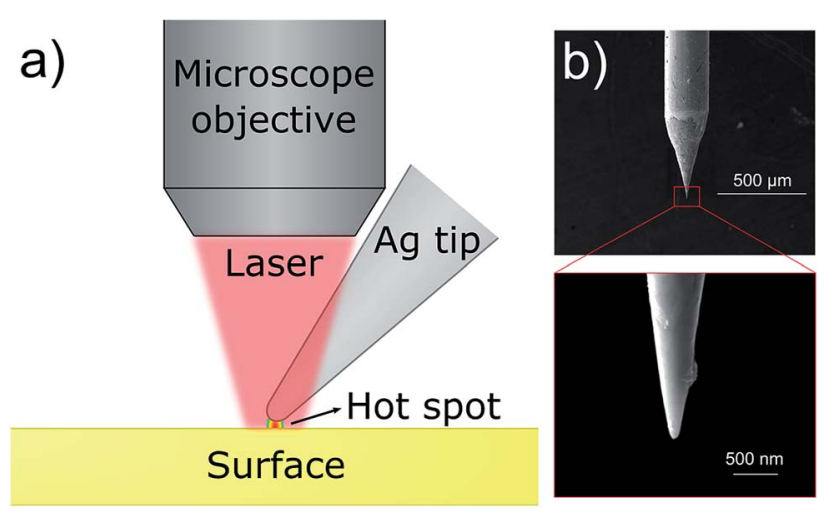

Fig. 2 (a) scheme of the top visual TERS apparatus employed in the measurements; (b) SEM image of a STM-TERS silver tip fabricated by the electrochemical etching. 

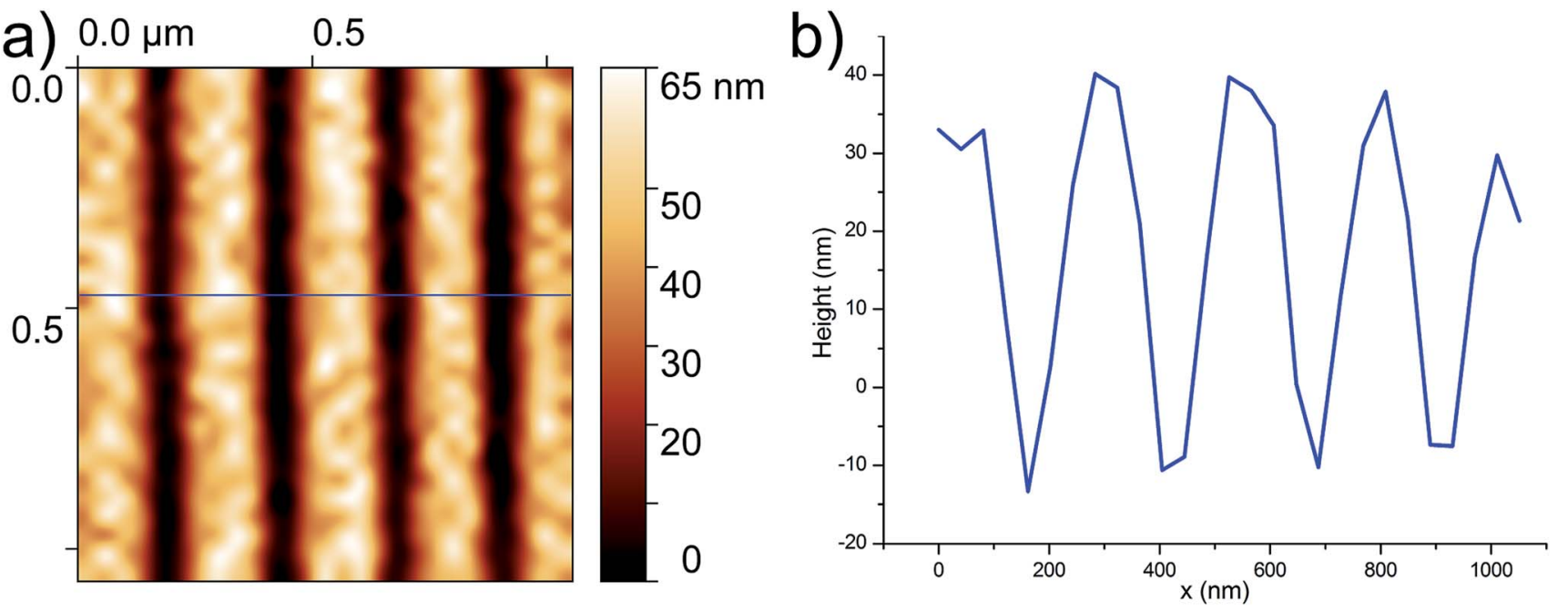

Fig. 3 AFM image (a) of a PMMA mask exposed to the ideal EBL dose of $670 \mu \mathrm{C} \mathrm{cm}^{-2}$, measured after development: the gold surface underneath the mask is exposed, yet the lines are still separate; a line profile taken on the blue line is reported in (b).

a circular Au cathode. ${ }^{23-25}$ This mechanism was electronically controlled by a homemade feedback circuit that automatically cut the voltage after the wire drop-off and the tips were formed, standardising the procedure to make it operator-independent in order to obtain apices whose curvature radii were centred around $40 \mathrm{~nm}$, with a dispersion of $10 \mathrm{~nm}$. AFM measurements were performed using the Ntegra SPM, in semi-contact mode in order not to modify the structure of the PMMA, using uncoated NT-MDT VIT-P top visual Si probes (resonant frequency $\sim 300$ $\mathrm{kHz}$ ). Confocal Raman measurements were carried out using the TERS apparatus without the tip mounted or with the out of focus tip when reported. TERS chemical intensity maps were calculated by measuring the area of the Lorentzian functions best fitting the peaks in the $\pm 50 \mathrm{~cm}^{-1}$ spectral region centred on the wavenumber of the signal on which the map is based, after background subtraction. The enhancement factors reported were calculated by measuring the height of the fitted Raman signal used for the calculation after background subtraction; when noise was considered, the standard deviation of the noise in the $1750-2250 \mathrm{~cm}^{-1}$ region (after background subtraction) was utilised.

\section{Results and discussion}

\section{Mask characterization}

Preliminary measurements with both STM and confocal Raman verified that the $\mathrm{Au} / \mathrm{Ti}$ evaporated surfaces are fully conductive and completely Raman inactive after thiophenol deposition. AFM tests and following characterizations also demonstrated that these substrates have a low roughness (ISO 4287 parameters for surface roughness: $S_{\mathrm{q}}=2.3 \mathrm{~nm}, S_{\mathrm{a}}=1.8 \mathrm{~nm}$ ). Moreover, they are robust enough to withstand vigorous AFM measurements in full contact, and mask deposition and lift-off by sonication. While it is possible to draw any kind of patterns onto the mask, simple and regular geometries are desirable for its use as a calibration standard. ${ }^{26,27}$ Repeating long, straight, parallel lines in a known direction respectively to the Si wafer were chosen in this study. Different EBL doses and varied line spacing were employed in order to establish the appropriate conditions for proper exposure, avoiding incomplete development due to insufficient doses, as well as averting overexposure and distortion/merging of pattern elements by determining the minimum line distance at which distinct features subsist when doses adequate for full exposure are employed.

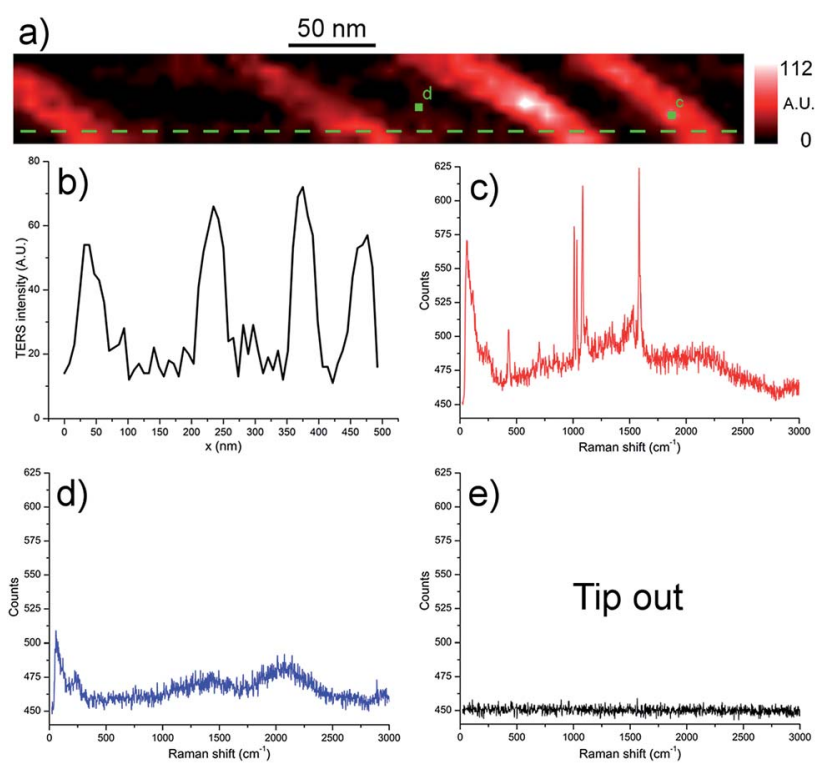

Fig. 4 STM-TERS chemical map (a), without corrections, based on the $1074 \mathrm{~cm}^{-1}$ Raman peak, of a nanopatterned thiophenol surface, obtained with $0.25 \mathrm{~mW}$ laser power and $0.5 \mathrm{~s}$ per pixel integration time (pixel size $\sim 7.5 \mathrm{~nm}$ ), and a line profile based on this signal (b), taken on the dashed line in (a). Raman spectra corresponding to the map are reported: a TERS spectrum on a SAM-covered area (c); a TERS spectrum corresponding to a bare gold pixel (d); a confocal Raman spectrum of the sample, measured with the tip retracted from the surface (e). The marks on the map indicate the pixels where the spectra (c) and (d) were taken. 


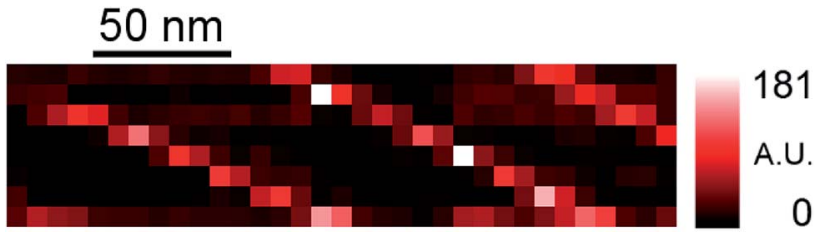

Fig. 5 STM-TERS raw chemical map of the $1074 \mathrm{~cm}^{-1}$ thiophenol peak on a nanopatterned substrate obtained from a partially underexposed map in order to reduce the pattern thickness below the beam diameter of the EBL. It was acquired with $0.25 \mathrm{~mW}$ laser power and $0.5 \mathrm{~s}$ integration time per pixel (pixel size $\sim 7.5 \mathrm{~nm}$ ).

After development with MIBK, the samples were measured with semi-contact AFM. In Fig. 3, an AFM map of the optimised mask is shown; since the mask height is $50 \mathrm{~nm}$, trench depths below this value are to be considered underdeveloped, since the $\mathrm{Au}$ surface below is necessarily covered by PMMA. After several dose experiments a dose of $670 \mu \mathrm{C} \mathrm{cm}^{-2}$ has been chosen as optimal for the fabrication of a reliable mask with predictable bare $\mathrm{Au}$ areas for subsequent thiol chemically selective bonding.

\section{TERS imaging}

TERS chemical imaging in STM mode was performed on the finalised samples in order to verify the presence and the integrity of the monolayers, and that the masks were completely removed by the treatment. This was proven by the fact that no PMMA signal was measured on the surfaces. Moreover it was verified that the portions of the surfaces that were supposed to remain pristine (because of the mask coverage and subsequent lift-off) were actually free of thiols, and that the molecules had not diffused onto them instead.

In Fig. 4, a TERS chemical map of one of the thiophenol patterned surfaces is shown, the red colour representing the $1074 \mathrm{~cm}^{-1}$ peak intensity $\left(\nu_{\mathrm{CC}}+\delta_{\mathrm{CH}}\right) .{ }^{17}$ Very recognisable parallel lines, corresponding to the spatial features previously measured on the PMMA masks, are clearly visible, and interspersed with zones with near zero intensity. An investigation of the corresponding spectra shows that no chemical was detected on these black areas, while pure benzenethiol spectra are registered on the lines corresponding to the organic pattern.

In order to test the limits of the our sample production process, a thinner pattern was drawn and a grating of thiophenol with lines roughly $8 \mathrm{~nm}$ wide were obtained. The resulting TERS chemical map of the $1074 \mathrm{~cm}^{-1}$ Raman shift can be seen in Fig. 5: lines with width of $(9 \pm 4)$ nm were measured (the line width is defined here as the mean and standard deviation of the FWHMs of the Gaussian interpolations of several intensity profiles along the scans). The map was measured with a $7.5 \mathrm{~nm}$ pixel size: by the Shannon-Nyquist theorem, faithful sampling cannot be performed at a rate lower than the Nyquist rate (i.e. half the lateral dimensions of the features, or about
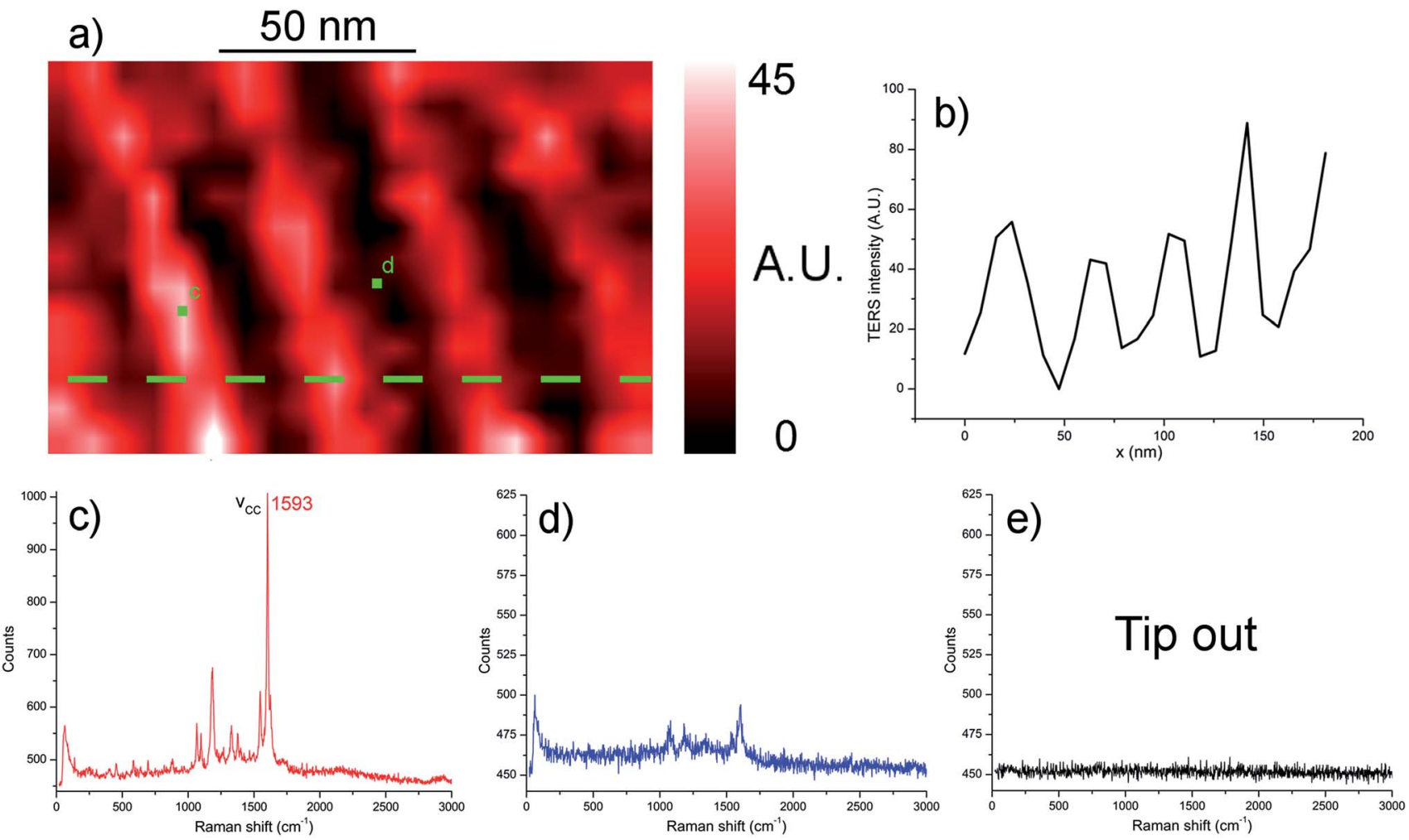

Fig. 6 STM-TERS chemical image (a), without corrections of a MMC-patterned sample, based on the $1593 \mathrm{~cm}^{-1} \mathrm{C}=\mathrm{C}$ stretching mode. The acquisition conditions are $0.25 \mathrm{~mW}$ laser power and $0.5 \mathrm{~s}$ per pixel as exposure time, step size $\sim 8 \mathrm{~nm}$. A line profile based on the intensity of this peak over the dashed line is shown in (b). Raman spectra of the sample with the same conditions are also reported: a TERS spectrum of the SAM (c); a spectrum of an area previously covered by the PMMA mask (d); a confocal Raman spectrum of the sample after the retraction of the tip (e). Marks on (a) correspond to the points where the spectra (c) and (d) were measured. 
$16 \mathrm{~nm}$ in this case), hence the reconstructed lines are prone to strong aliasing.

Thiophenol is a widely used molecule in plasmon-enhanced Raman studies, ${ }^{\mathbf{1 7}}$ but the aforementioned patterning approach was investigated for the application to a different thiol as well. The chosen molecule was 7-mercapto-4-methylcoumarine (MMC), a relatively unexplored molecule in TERS that was found to yield much more intense plasmon-enhanced Raman signals than thiophenol. In Fig. 6, a TERS chemical map of the nanopatterned MMC sample after mask lift-off is shown, coloured based on the intensity of the $1593 \mathrm{~cm}^{-1}$ MMC $\mathrm{C}=\mathrm{C}$ stretching band. ${ }^{28,29}$ Nevertheless, this map exhibits feeble MMC signals in areas that were covered during the molecule deposition step of the fabrication process, suggesting lesser stability of the monolayer during the mask lift-off process, or because of tip contamination during the TERS measurement, or both.

Beside the spatial distribution, the Enhancement Factor (EF) can be estimated too from the TERS maps. The EF is often defined $^{30,31}$ as the factor by which the Raman effect is amplified by the tip in the effective hotspot area, hence:

$$
\mathrm{EF}=\frac{I_{\mathrm{TI}}-I_{\mathrm{TO}}}{I_{\mathrm{TO}}} \frac{A_{\mathrm{FF}}}{A_{\mathrm{NF}}}
$$

where $I_{\mathrm{TI}}$ is the signal intensity measured when the tip is in the vicinity of the sample, $I_{\mathrm{TO}}$ represents the Raman intensity measured when the tip is away from the sample, while $A_{\mathrm{NF}}$ and $A_{\mathrm{FF}}$ stand for the volume investigated by the hotspot (in the present study approximated as a sphere with a radius equal to the tip apex radius) and the confocal volume respectively, to take into consideration the different number of molecules from which the signal arises. In this study, given the assumption of a perfect SAM on a flat surface, the volumes ratio is the same as the ratio of the areas resulting from the intersection of said volumes with the monolayer plane. However, because of the very small quantity of analyte in the few $\mu \mathrm{m}^{2}$ of investigated SAM area, no thiol Raman spectrum is detectable: therefore, an accurate estimate of the actual EF cannot be achieved; nevertheless, its lower limit can be determined if $N_{\mathrm{TO}}$, defined as three times the standard deviation of the noise when the tip is far from the sample (a conventionally accepted threshold for the definition of detection limit in spectra ${ }^{32}$ ), is treated as the maximum possible intensity for the TO spectrum (i.e. the maximum undetectable signal):

$$
\mathrm{EF}>\frac{I_{\mathrm{TI}}-N_{\mathrm{TO}}}{N_{\mathrm{TO}}} \frac{A_{\mathrm{FF}}}{A_{\mathrm{NF}}}
$$
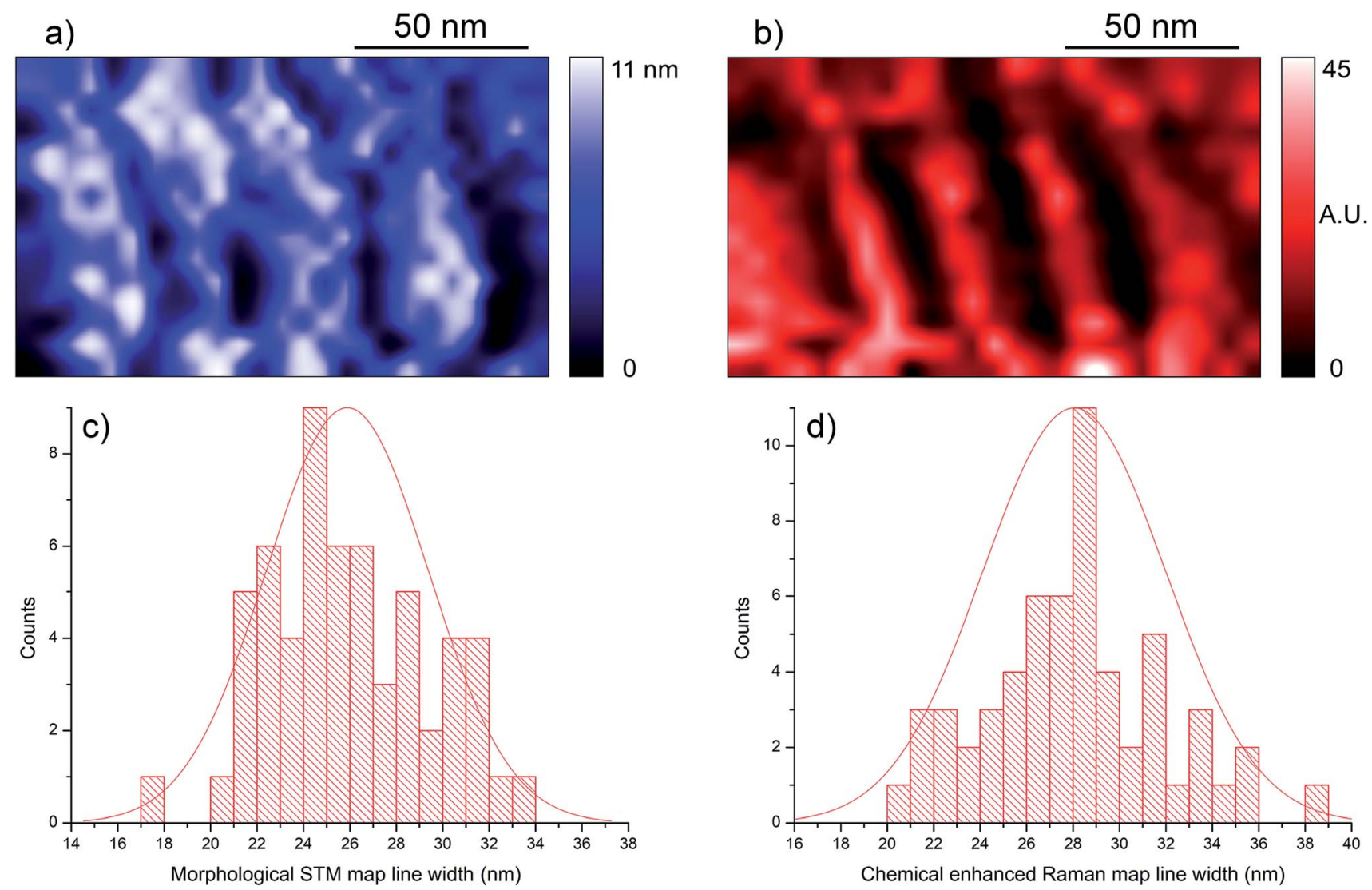

Fig. 7 Comparison of topographic (a) and chemical (b) images of an area of the proposed sample, acquired concurrently by STM-TERS. The image was acquired with a tunneling current feedback of $0.5 \mathrm{nA}$ at $0.1 \mathrm{~V}$, a laser power of $0.25 \mathrm{~mW}, 0.5 \mathrm{~s}$ per pixel ( $~ 8 \mathrm{~nm}$ pixel size). The chemical image is based on the intensity of the $1593 \mathrm{~cm}^{-1} \mathrm{MMC}$ peak. (c and d): statistical analysis of the thiol lines width measured in the top maps and superimposed Gaussian distribution curves with the samples means and standard deviations as parameters. Line width in the topographic map: mean $\mu=25.9 \mathrm{~nm}$ and standard deviation $\sigma=3.5 \mathrm{~nm}$. Line width in the chemical map: mean $\mu=28.1 \mathrm{~nm}$ and standard deviation $\sigma=3.9 \mathrm{~nm}$. 
The lower EF limits were found to be EF $>(2.2 \pm 0.5) \times 10^{7}$ for thiophenol, and EF $>(1.0 \pm 0.2) \times 10^{8}$ in the case of MMC. In surface- and tip-enhanced Raman spectroscopy, it is common to be unable to resolve a non-enhanced signal from the confocal Raman measurements. A formula for the EF estimation in such a case, adapted from ${ }^{29}$ for TERS, is the following:

$$
\mathrm{EF}=\frac{I_{\mathrm{TI}}-I_{\mathrm{TO}}}{1+I_{\mathrm{TO}}} \frac{A_{\mathrm{FF}}}{A_{\mathrm{NF}}}
$$

from which an EF of $(1.7 \pm 0.4) \times 10^{8}$ for thiophenol, and an EF of $(7.4 \pm 1.6) \times 10^{8}$ for MMC is calculated.

A comparison between the TERS chemical and topographic images on these substrates can be carried out in order to extract dimensional information about the probe chemical capabilities. The aforementioned line patterns are quite practical for this purpose, because measuring the lines width in both maps is straightforward, and it generates a large sample size, given that each scan can be considered an independent measurement. In Fig. 7, the topographic and chemical TERS maps of the same sample area are shown, with the corresponding statistical analysis. While the sample is flat and the SAM lines have modest thickness, quite noticeable trenches are visible in the topographic map. This is due to the difference in conductivity between the naked, previously masked gold areas and the SAM areas on gold: in a constant current STM map such as this, less conductive areas on a flat sample are registered as spatially lower because of the tip movement necessary to maintain the feedback. Each thiol line and tip scan were considered as independent measurements of the same quantity, namely the line width defined as the distance between the two edges at $10 \%$ of the maximum intensity (in the chemical map) or depth (for the STM map). The lines have a mean width of $\mu=25.9 \mathrm{~nm}$ with a standard deviation of $\sigma=3.5 \mathrm{~nm}$ in the STM measurement, and a mean of $\mu=28.1 \mathrm{~nm}$ with $\sigma=3.9 \mathrm{~nm}$ standard deviation in the chemical image. This indicates a larger line width in the chemical map, most likely caused by the larger effective probe size: while a probe used in STM can be approximated to have apex dimensions of a few $\AA$, with a similar interaction radius with the surface, ${ }^{33}$ TERS hotspots are significantly broader. An SPM spatial measurement yields the convolution of the target feature with the tip shape and size, therefore these findings are consistent with the expected effects.

\section{Conclusions}

In the present work, a candidate reference sample for calibration of TERS spatial resolution was proposed, consisting of a flat gold surface with customizable thiol monolayer nanopatterns; this sample is expected to be suitable for both STM and forcebased TERS feedbacks (AFM, shear force microscopy). The ideal conditions for its fabrication were found by AFM measurements during production, and the final product was tested with TERS mapping in STM mode. To test the extent of its customizability, both a proven TERS model molecule, thiophenol, and a novel thiol for TERS measurements, 7-mercapto4-methylcoumarin, were used for the fabrication of the substrates. The benzenethiol surfaces were found to be very stable, and the expected pattern was measured with a quite good Raman signal. The MMC monolayer was found to have a much greater TERS signal with respect to the thiophenol SAM: the former molecule was measured to have an EF lower limit more than 4 times larger than that of the latter; however, the measurements suggest that the stability of the MMC system is somewhat lower than those of the thiophenol SAM. A numerical comparison between the topographic and chemical components of a TERS image was performed as well. The low dispersion of the thiol lines in both the physical and chemical image of the nanopatterned monolayer, as well as the accordance of these measurements to the predictions, indicate that this substrate is a viable candidate reference sample for the calibration and comparison of TERS spatial resolution.

\section{Conflicts of interest}

There are no conflicts to declare.

\section{Acknowledgements}

The project 15HLT01 MetvBadBugs has received funding from the EMPIR programme co-financed by the Participating States and from the European Union's Horizon 2020 research and innovation programme.

\section{References}

1 S. Kawata and V. M. Shalaev. Tip enhancement, Elsevier, 2011. 2 R. M. Stöckle, Y. D. Su, V. Deckert and R. Zenobi, Nanoscale chemical analysis by tip-enhanced Raman spectroscopy, Chem. Phys. Lett., 2000, 318, 131-136.

3 N. Hayazawa, Y. Inouye, Z. Sekkat and S. Kawata, Metallized tip amplification of near-field Raman scattering, Opt. Commun., 2000, 183, 333-336.

4 N. Lee, R. D. Hartschuh, D. Mehtani, A. Kisliuk, J. F. Maguire, M. Green, M. D. Foster and A. P. Sokolov, High contrast scanning nano-Raman spectroscopy of silicon, J. Raman Spectrosc., 2007, 38, 789-796.

5 R. Zhang, Y. Zhang, Z. C. Dong, S. Jiang, C. Zhang, L. G. Chen, L. Zhang, Y. Lia, J. Aizpurua, Y. Luo, J. L. Yang and J. G. Hou, Chemical mapping of a single molecule by plasmon-enhanced Raman scattering, Nature, 2013, 498, 82-86.

6 M. S. Anderson, Locally enhanced Raman spectroscopy with an atomic force microscope, Appl. Phys. Lett., 2000, 76, 31303132.

7 N. Kumar, S. J. Spencer, D. Imbraguglio, A. M. Rossi, A. J. Wain, B. M. Weckhuysenb and D. Roy, Extending the plasmonic lifetime of tip-enhanced Raman spectroscopy probes, Phys. Chem. Chem. Phys., 2016, 18, 13710-13716.

8 C. C. Neacsu, J. Dreyer, N. Behr and M. B. Raschke, Scanningprobe Raman spectroscopy with single-molecule sensitivity, Phys. Rev. B: Condens. Matter Mater. Phys., 2006, 73, 193406.

9 C. Zhang, B. Chen and Z. Li, Optical Origin of Subnanometer Resolution in Tip-Enhanced Raman Mapping, J. Phys. Chem. C, 2015, 119, 11858-11871. 
10 F. Latorre, S. Kupfer, T. Bocklitz, D. Kinzel, S. Trautmann, S. Gräfea and V. Deckert, Spatial resolution of tipenhanced Raman spectroscopy - DFT assessment of the chemical effect, Nanoscale, 2016, 8, 10229-10239.

11 X. Wang, S.-C. Huang, T.-X. Huang, H.-S. Su, J.-H. Zhong, Z.-C. Zeng, M.-H. Li and B. Ren, Tip-enhanced Raman spectroscopy for surfaces and interfaces, Chem. Soc. Rev., 2017, 46, 4020-4041.

12 Y. Fang, Z. Zhang and M. Sun, High vacuum tip-enhanced Raman spectroscope based on a scanning tunneling microscope, Rev. Sci. Instrum., 2016, 87, 033104.

13 Z. Zhang., S. Sheng, R. Wang and M. Sun, Tip-Enhanced Raman Spectroscopy, Anal. Chem., 2016, 88, 9328-9346.

14 Z. Zeng, S. Huang, D. Wu, L. Meng, M. Li, T. Huang, J. Zhong, X. Wang, Z. Yang and B. Ren, Electrochemical Tip-Enhanced Raman Spectroscopy, J. Am. Chem. Soc., 2015, 137, 1192811931.

15 V. Korpelainen, Traceability for nanometre scale measurements: atomic force microscopes in dimensional nanometrology, PhD thesis, Department of Physics, University of Helsinki, Finland, 2014.

16 R. Breil, T. Fries, J. Garnaes, J. Haycocks, D. Hußser, J. Joergensen, W. Kautek, L. Koenders, N. Kofod, K. R. Koops, R. Korntner, B. Lindner, W. Mirandeé, A. Neubauer, J. Peltonen, G. B. Picotto, M. Pisani, H. Rothe, M. Sahre, M. Stedman and G. Wilkening, Intercomparison of scanning probe microscopes, Precis. Eng., 2002, 26, 296-305.

17 C. Blum, L. Opilik, J. M. Atkin, K. Braun, S. B. Kämmer, V. Kravtsov, N. Kumar, S. Lemeshko, J. Li, K. Luszcz, T. Maleki, A. J. Meixner, S. Minne, M. B. Raschke, B. Ren, J. Rogalski, D. Roy, B. Stephanidis, X. Wang, D. Zhang, J. Zhong and R. Zenobi, Tip-enhanced Raman spectroscopy-an interlaboratory reproducibility and comparison study, J. Raman Spectrosc., 2014, 45, 22-31.

18 J. Stadler, B. Oswald, T. Schmid and R. Zenobi, Characterizing unusual metal substrates for gap-mode tipenhanced Raman spectroscopy, J. Raman Spectrosc., 2013, 44, 227-233.

19 G. Picardi, M. Chaigneau and R. Ossikovski, High resolution probing of multi wall carbon nanotubes by tip enhanced Raman spectroscopy in gap-mode, Chem. Phys. Lett., 2009, 469, 161-165.

20 C. D. Bain and G. M. Whitesides, Formation of monolayers by the coadsorption of thiols on gold: variation in the length of the alkyl chain, J. Am. Chem. Soc., 1989, 111, 7164-7175.

21 J. C. Love, L. A. Estroff, J. K. Kriebel, R. G. Nuzzo and G. M. Whitesides, Self-Assembled Monolayers of Thiolates on Metals as a Form of Nanotechnology, Chem. Rev., 2005, 105, 1103-1170.

22 J. Stadler, T. Schmid and R. Zenobi, Nanoscale Chemical Imaging Using Top-Illumination Tip-Enhanced Raman Spectroscopy, Nano Lett., 2010, 10, 4514-4520.

23 W. Zhang, B. S. Yeo, T. Schmid and R. Zenobi, Single Molecule Tip-Enhanced Raman Spectroscopy with Silver Tips, J. Phys. Chem. C, 2007, 111, 1733-1738.

24 S. S. Kharintsev, A. I. Noskov, G. G. Hoffmann and J. Loos, Near-field optical taper antennas fabricated with a highly replicable ac electrochemical etching method, Nanotechnology, 2011, 22, 025202.

25 J. S. Lloyd, A. Williams, R. H. Rickman, A. McCowen and P. R. Dunstan, Reproducible electrochemical etching of silver probes with a radius of curvature of $20 \mathrm{~nm}$ for tipenhanced Raman applications, Appl. Phys. Lett., 2011, 99, 143108.

26 R. Koops, M. van Veghel and A. van de Nes, A dedicated calibration standard for nanoscale areal surface texture measurements, Microelectron. Eng., 2015, 141, 250-255.

27 F. Stöhr, J. Michael-Lindhard, H. Simons, H. F. Poulsen, J. Hübner, O. Hansen, J. Garnaes and F. Jensen, Threedimensional nanometrology of microstructures by replica molding and large-range atomic force microscopy, Microelectron. Eng., 2015, 141, 6-11.

28 A. Taglietti, Y. A. Diaz Fernandez, P. Galinetto, P. Grisoli, C. Milanese and P. Pallavicini, Mixing thiols on the surface of silver nanoparticles: preserving antibacterial properties while introducing SERS activity, J. Nanopart. Res., 2013, 15, 2047-2052.

29 S. A. Kara, A. Keffous, A. M. Giovannozzi, A. M. Rossi, E. Cara, L. D'Ortenzi, K. Sparnacci, L. Boarino, N. Gabouze and S. Soukane, Fabrication of flexible silicon nanowires by self-assembled metal assisted chemical etching for surface enhanced Raman spectroscopy, RSC Adv., 2016, 6, 93649-93659.

30 N. Kumar, A. Rae and D. Roy, Accurate measurement of enhancement factor in tip-enhanced Raman spectroscopy through elimination of far-field artefacts, Appl. Phys. Lett., 2014, 104, 123106.

31 D. Roy, J. Wang and C. Williams, Novel methodology for estimating the enhancement factor for tip-enhanced Raman spectroscopy, J. Appl. Phys., 2009, 105, 013530.

32 G. L. Long and J. D. Winefordner, Limit of detection. A closer look at the IUPAC definition, Anal. Chem., 1983, 55, 712A724A.

33 V. L. Mironov, Fundamentals of Scanning Probe Microscopy, The Russian Academy of Sciences, 2004. 